\title{
A model for prediction of microbial disease burden of water supply options in Bangladesh
}

\author{
M. Feroze Ahmed \\ Stamford University Bangladesh, 51 Siddeswari Road, Dhaka 1217, Bangladesh
}

Received 11 November 2014/Accepted 29 November 2014

\begin{abstract}
Infectious diseases caused by microbial pathogens are most common and widespread health risks are associated with drinking water. This paper presents a model for prediction of disease burden for ingestion of microbial pathogens through drinking water in rural Bangladesh. The concentration of indicator organisms (Thermotolerant coliform or Escherichia coli) in cfu/100ml were taken as input in the model and estimated disease burden in $\mu \mathrm{DALYs}$ with some relevant statistical parameters are obtained as output. The model has been found useful in quantifying relative disease burden of microbial pathogens, comparing safety of different water supply options and delineating the interventions needed to reduce disease burden from drinking water.
\end{abstract}

Key words: DALYs; Disease burden; Pathogens; Risk assessment

Safe, adequate and accessible supplies of water, together with proper sanitation are basic needs and essential components of primary health care. Inadequate provision of safe drinking water and sanitation are directly and indirectly related to the communicable diseases, health risk, poor health and poverty. The most serious water contamination problem in developing countries is the fecal pollution originated from indiscriminate defecation and inadequate sanitation facilities (1-3). Globally 1.8 million people die every year of diarrhoeal diseases including cholera and $88 \%$ of diarrhoeal diseases are attributed to unsafe water supply and sanitation (1,4). An estimated 99.7 million Disability-Adjusted Life Years (DALYs) are contributed by diarrhoeal diseases to global disease burden. In Bangladesh, the estimated diarrhoeal disease burden is 1.8 million DALYs (5).

The estimation of disease burden of a water supply is an important measure of the risk to health associated with drinking water (1). It is a principal method of establishing health-based target within the Water Safety Framework promoted by World Health Organization (6). The health outcome target is primarily applicable to microbial hazards in both developed and developing countries. Health outcome results may be employed directly as a basis to define water quality targets or may provide the basis for development of performance target (6). DALY is a good method of comparing the different

${ }^{\star}$ Corresponding Author: Mailing address. Prof. Dr. M. Feroze Ahmed, Vice Chancellor, Stamford University Bangladesh, 51 Siddeswari Road, Dhaka 1217, Bangladesh; E-mail: vc@stamforduniversity.ac.bd. health outcomes for a range of diseases and injuries in a common metric and allows different hazards to be compared when assessing the importance of their impact on health. Disease burden expressed in DALYs also provides a good basis for analysis of economic benefit/burden of improvement/deterioration of microbial quality of water supplies.

The main purpose of this paper is to assess the disease burden from microbial pathogens in drinking water. The use of quantitative microbial risk health assessment (QMHRA) is an emerging tool to support decision-making in developing countries. The benefit of quantitative risk assessment is that it allows comparisons to be made among different technologies based on often relatively easily acquired input data. It is a mathematical and logical expression of current knowledge informed by the available scientific and epidemiological evidence. As such, QMHRA provides a "current best estimate" or "bestsupported estimate" of disease burdens associated with exposures. Therefore, QMHRA is often the best available low-cost technique to estimate disease burdens using updated available information. The use of QMHRA is valuable where a disease burden estimate is used as an input to decision-making which might otherwise only be made on the basis of water quality data in the absence of a health-related interpretation.

\section{METHODOLOGY}

The Model Development. To support estimation of the disease burden associated with rural water supplies in Bangladesh, a model based quantitative health risk assessment was developed. This was based on the accepted risk assessment process of problem formulation and hazard identification, exposure assessment, doseresponse assessment and risk characterization. The problem formulation was the 


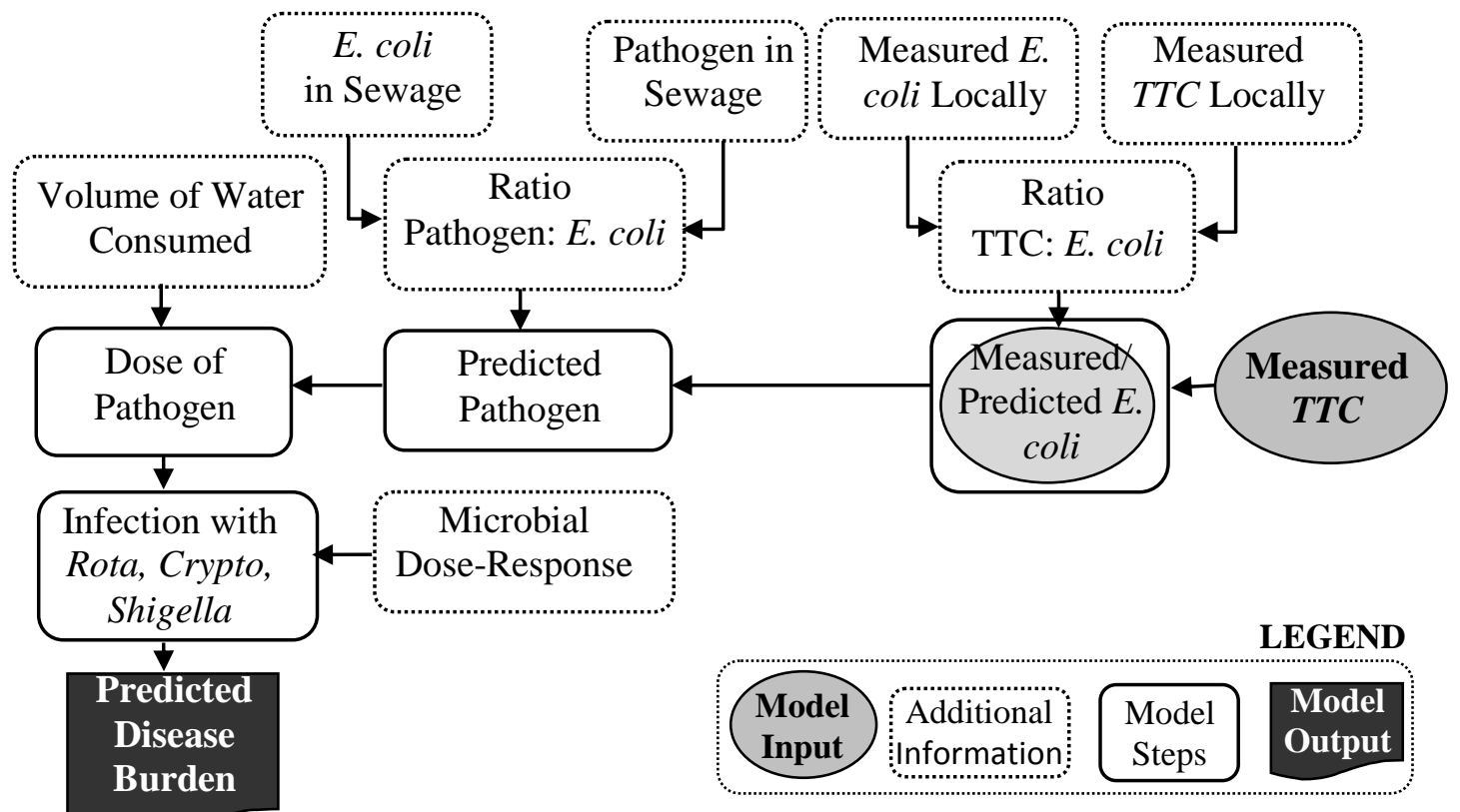

FIG. 1. The architecture of the model for prediction of disease burden

degree of public risk associated with water supplies installed in rural areas. The main hazards were identified as pathogens and arsenic. The exposure assessment involved estimating the size of population exposed and the level of hazard to which they were likely to be exposed. The dose response assessment provided the basis for estimating the health implications of the modelled exposure. Risk characterisation normally involves comparing the outcomes of the risk assessment model to some benchmark level; and/or comparing a range of modelled scenarios to select the most favourable. In this case comparisons were made to the suggested reference level of $10^{-6}$ DALYs per infection suggested by WHO (6).

The architecture of the disease burden estimation by QMHRA is presented in Figure 1. The model used faecal indicator organisms instead of reference pathogens because of its widespread use in developing countries as indicator of microbial pollution. Measurement of concentration of reference pathogens in the control of water quality is difficult and not in practice in developing countries. The process starts with the measured concentrations of thermotolerant coliforms (TTC) (or E. coli where direct analysis is performed) in colony forming units (cfu) per $100 \mathrm{ml}$. The process ends with predicted disease burden estimates in units of Disability Adjusted Life Years (DALYs) for each risk endpoint.

In the microbial stream of the process, the local ratio of $E$. coli and TTC are used to estimate the concentration of $E$. coli. The ratio of pathogen and $E$. coli in sewage based on results from long-term monitoring in Australia is used to estimate the possible concentration of pathogens and volume of unboiled water consumed. The dose-response relationships for the model reference pathogens were based on reported human-feeding-trial (HFT) data. For "virus" the rotavirus model of Gerba et al. (7) citing the HFT of Ward et al. (8) was applied since it is an endemic and routinely surveyed bacterial infection in Bangladesh (9). The beta-Poisson distribution was selected because as noted by Teunis et al. (10) it has been corroborated and widely used since being proposed by Gerba et al. (7). For the "bacterium" Shigella dysenteriae model of Holcomb et al. (11) citing the HFT of Levine et al. (12) was applied, which is an endemic and routinely surveyed bacterial infection in Bangladesh (9). The Weibull-gamma relationship was selected because it provided the smallest over-estimate at below-threshold doses from the acceptable-fitting inflection models. For "protozoan" the "unknown strain" model for Cryptosporidium parvum of Messner et al. (13) citing the HFTs of DuPont et al. (14), Okhuysen et al. (15) and Chappell et al. (16) was applied. Cryptosporidium was selected for the protozoan model reference pathogen as it was environmentally more mobile, persistent and infectious pathogen than the alternatives Giardia and Entameoba.

The microbial dose-response functions used to predict probability of infection are shown in Table 1 . The daily dose of pathogens consumed was converted to a daily probability of infection according to these dose-response relationships to give an infection endpoint prediction for each pathogen. The daily probability of infection was converted to an annual incidence of infection as described by Teunis et al. (10), which provided the input to the DALY calculation. The disease burden estimates described by Havelaar and Melse (17) were selected for viral, protozoal and bacterial disease.

To determine the disease burden, severity weights given in Murray and Lopez (18) are multiplied by duration of the effects to estimate the DALYs per case. It is then multiplied by the number of people affected to give a population disease burden. DALYs are, therefore, useful when considering overall public health priorities or where comparisons are being made between two different water supply options where a number of hazards may be present. The use of DALYs in decisionmaking has been promoted by WHO following the global burden of disease analysis of Murray and Lopez (18) and DALYs are now widely used for public health reporting and decision-making by health agencies worldwide. The use of DALYs in water-related scenarios has been promoted by WHO $(6,17)$.

The User Interface. A spreadsheet model has been developed to simplify the computation in the different stages of the model. The user interface of the model with input and output is shown in Figure 2. Thermotolerant coliform (TTC) or E. coli in cfu/100 ml is the main input in the model. The median value of a series of microbial monitoring data of a drinking water source, and if desired $95 \%$ with minimum and maximum values of the data series can be entered to obtain normalized disease burden. If $E$. coli concentrations are entered, the values are directly used by the model. If thermotolerant coliform concentrations are entered, the model estimates the proportion that is E. coli. A "data tool" work sheet has been provided to find out $95 \%$ ile, median, minimum and maximum values of a data series. The geometric mean can be used in place of the median and usually this value is very close to the median. However, the common presence of zero values (a value below detection limit of the equipment used) or one or few abnormally high value(s) prevents simple calculation of the geometric mean. If the $95 \%$ ile value is entered, the spreadsheet uses the difference between the median and $95 \%$ to estimate the parameters of a lognormal probability density function for the dataset. The variability in the dataset is multiplicatively propagated through the model to contribute to the upper and lower limits of the $90 \%$ confidence interval, which is then reported as an output.

The model allows the user to modify the measured volume of water consumed by the population of interest. Both a median and $95 \%$ values of water consumed can be entered. The model also allows the user to modify the predicted life expectancy of birth for the population of interest but it also requires the change of population distribution of the country concerned. Only changing the value for life expectancy at birth will capture part of the effect on disease burdens but not all of the effect.

The model outputs as shown in Figure 2 are total disease burden in $\mu$ DALY per person-year with a breakdown of $\mu$ DALY contributed by different types of microbes. The median value, upper and lower limits of $90 \%$ confidence interval (CI) when $95 \%$ ile value is entered and the $\mu \mathrm{DALY}$ rages when minimum and maximum values are entered. Outputs from the model can be used to compare microbial DALYs and relative DALYs contributed by different species of 
TABLE 1. Microbial dose response functions and parameters used for estimation of probability of infection

\begin{tabular}{|c|c|c|}
\hline Pathogens & Equations & Parameter values \\
\hline $\begin{array}{c}\text { Virus } \\
\text { (Rotavirus) }\end{array}$ & $\begin{array}{c}\rho \text { inf }=1-(1+\delta / \beta)^{-\alpha} \\
\text { beta-Poisson } \\
\text { Gerba et al. (7) citing Ward et al. (8) }\end{array}$ & $\begin{array}{c}\rho \text { inf }=\text { probability of infection } \\
\delta=\text { dose } \\
\alpha=0.26 \\
\beta=0.42\end{array}$ \\
\hline $\begin{array}{l}\text { Bacterium } \\
\text { (Shigella } \\
\text { dysenteriae) }\end{array}$ & $\begin{array}{l}\qquad \rho \text { inf }=1-\left(1+\delta^{x / \beta}\right)^{-\varepsilon} \\
\text { Weibull-gamma } \\
\text { Holcomb et al. (11) citing Levine et al. (12) }\end{array}$ & $\begin{array}{c}\rho \text { inf }=\text { probability of infection } \\
\delta=\text { dose } \\
\beta=22.5 \\
\varepsilon=0.25 \\
\chi=1.08\end{array}$ \\
\hline $\begin{array}{l}\text { Protozoan } \\
\text { (C. Parvum) }\end{array}$ & $\begin{array}{c}\qquad \inf =1-e^{1-(\delta / \kappa)} \\
\quad \text { Exponential } \\
\text { Messner et al. (13) citing DuPont et al. (14); Okhuysen et al. (15) and } \\
\text { Chappell et al. (16) }\end{array}$ & $\begin{array}{c}\rho \inf =\text { probability of infection } \\
\delta=\text { dose } \\
\kappa=35.2\end{array}$ \\
\hline
\end{tabular}

\section{Input / Output Worksheet}

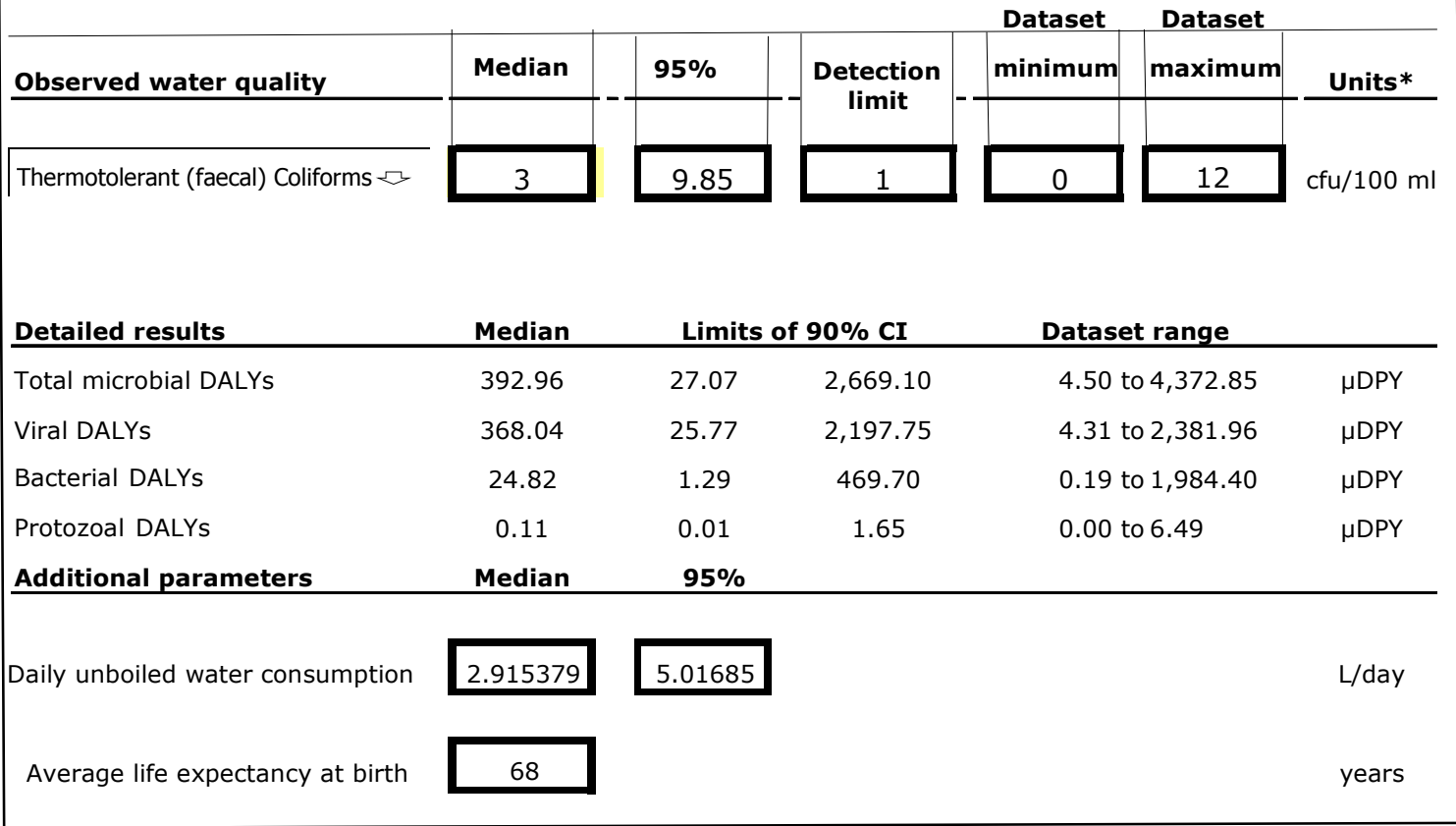

$\mathrm{cfu}=$ colony forming units); $\mathrm{py}=$ person/year

FIG. 2. The user interface of the model

microbes. The model also provides a graphical output of the results showing median and $90 \%$ confidence interval along with DALY scores of some selected guideline/standards values.

The model has been customized for Bangladesh conditions with data and assumptions as applicable in the country. The background levels of immunity to the viral, bacterial and protozoan reference pathogens were assumed to be relatively high due to the high background levels of disease borne by hygienerelated and other routes of transmission. However, these assumptions are tentative and are based on the opinion of local health sector professionals from WHO, UNICEF and ICDDR,B rather than the objective data. The probability of death per symptomatic case, the case fatality ratio (CFR), for the viral and bacterial pathogens were set at $0.23 \%$, a figure based on the 1991 Bangladesh census for hospitalized deaths from diarrhea. A median of 2.9 1/day with 95\%ile value of $5.0 \mathrm{~L} /$ day water consumption was used as input based on a study in hotter season in Bangladesh conducted by Watanabe et al. (19). Lifer expectancy in Bangladesh at birth of 68 years was used for both sexes (20).
Application of the Model. The presence of microbial pathogens in variable concentration in different water supply options made it difficult to compare the safety of water supplies using water quality data. So it was decided to evaluate the options by compiling water quality data of statistically representative water supply options under operating conditions in both wet and dry seasons and then transforming the water quality data into uniform DALY metric. The transformation of water quality into DALY required a lot of computation; the disease burden model was developed to make the transformation process easier. The model was applied to estimate disease burden of drinking water from Dug Well (DW), Shallow Tubewell (STW), Deep Tubewell (DTW), Pond Sand Filter (PSF) and Rainwater Harvesting (RWH) in Bangladesh. These are the common water supply technologies used in rural Bangladesh but the relative microbial safety of these water supply options is not known. Thermotolerant coliform (TTC) and E.coli counts of statistically representative samples from these drinking water sources across the country were made using standard method. The sampling method required analysis of water samples from 36 DTW, 36 DW, 42 RWHS and 42 PSF 


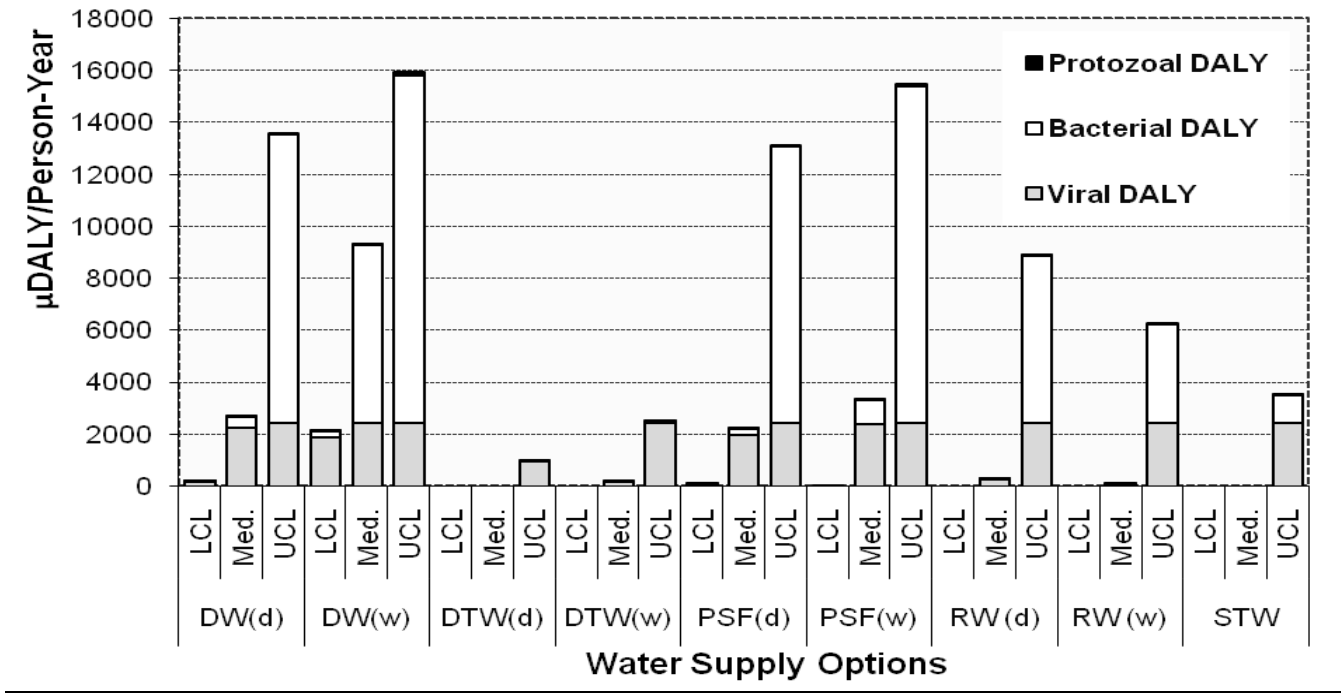

FIG. 3. Viral, bacterial and protozoal disease burden of water supply options

from 27 clusters in the country. Microbial quality of 24 shallow tubewells was also analyzed. Proper QA/QC was adopted in the analysis of all water samples. Both TTC and E.coli counts were made to establish a ratio of presence of these two types of indicator microorganisms in drinking water.

\section{RESULTS AND DISCUSSION}

In cases of all water supply options, the disease burdens contributed by rotaviruses, Shigella spp. and Cryptococcus spp. were estimated on the basis of measured TTC/E. coli which are expressed in DALY by using the model and presented in Figure 3. The model run at different concentrations of TTC or E. coli showed that viral and bacterial pathogens concentrations dominated the disease burden estimates for the microbial DALY results with crypto contributing relatively negligible risks to the total. At the lower TTC concentrations the viral disease burden was the most significant contributor and at higher TTC concentrations the bacterial disease burden began to dominate as the viral disease burden reached its saturation point. The greater proportions of the total DALYs attributed to each water supply technology at upper 95\% confidence limits (UCL) were microbial DALYs and those at median and lower 5\% confidence limits (LCL) are viral DALYs.

The LCL, median, UCL and $90 \%$ confidence interval of combined microbial DALYs of different water supply options are shown in Figure 4. The disease burdens of drinking water from STW, DTW and RWH are comparatively lower than those of DW and PSF. The $90 \%$ confidence intervals of all options are wide because of wider variations of microbial water quality. The lower 5 percentile disease burdens of water from STW, DTW, RWHS are quite low, even lower than the WHO reference level risk of $1 \mu$ DALY. The disease burdens of DW, DTW and PSF water increase in the wet season and those of water from RWHS increase in the dry season. The median risks of only STW and DTW waters in the dry season approached to the WHO reference level risk while median risks water from all other sources do not meet the reference level of risk. In general, the lowest risk options in terms of disease burden were the STW, DTW and RWH, although all indicated significantly high upper $95^{\text {th }}$ percentile microbial risk. Based the data collected in this study and data presented in other studies, it is clear that in overall terms STW and DTW offer the best option followed by RWH with regard to public health risks.

As stated above, the most useful ways in which the model can be used is to compare model outputs from one water supply scenario with another in cases where multiple health hazards are present. The objective of the QMHRA was to compare disease burdens predicted to be associated with different water supply options with one another to establish relative risk for each. In Bangladesh, the model was used as an effective tool to estimate disease burden of different water supply options $(21,22)$. Comparing the predicted disease burdens with one another it was possible to compare water supply options readily to make better-informed decisions about which options were likely to present the lowest relative disease burdens

The model provides an output as shown in Figure 4 to compare estimated disease burden of a water supply with the reference values. The most conservative reference value is the $1 \mu \mathrm{DALY} /$ person-year (DPY) which is used as the default point of reference for deriving guideline values for microbial hazards (6). Importantly, this WHO reference value is a 'per hazard' value, not a water supply system value. Clearly, if many microbial hazards are present at their guideline values, and each contributes 1 $\mu \mathrm{DPY}$ in disease burden, then the sum of the many health 


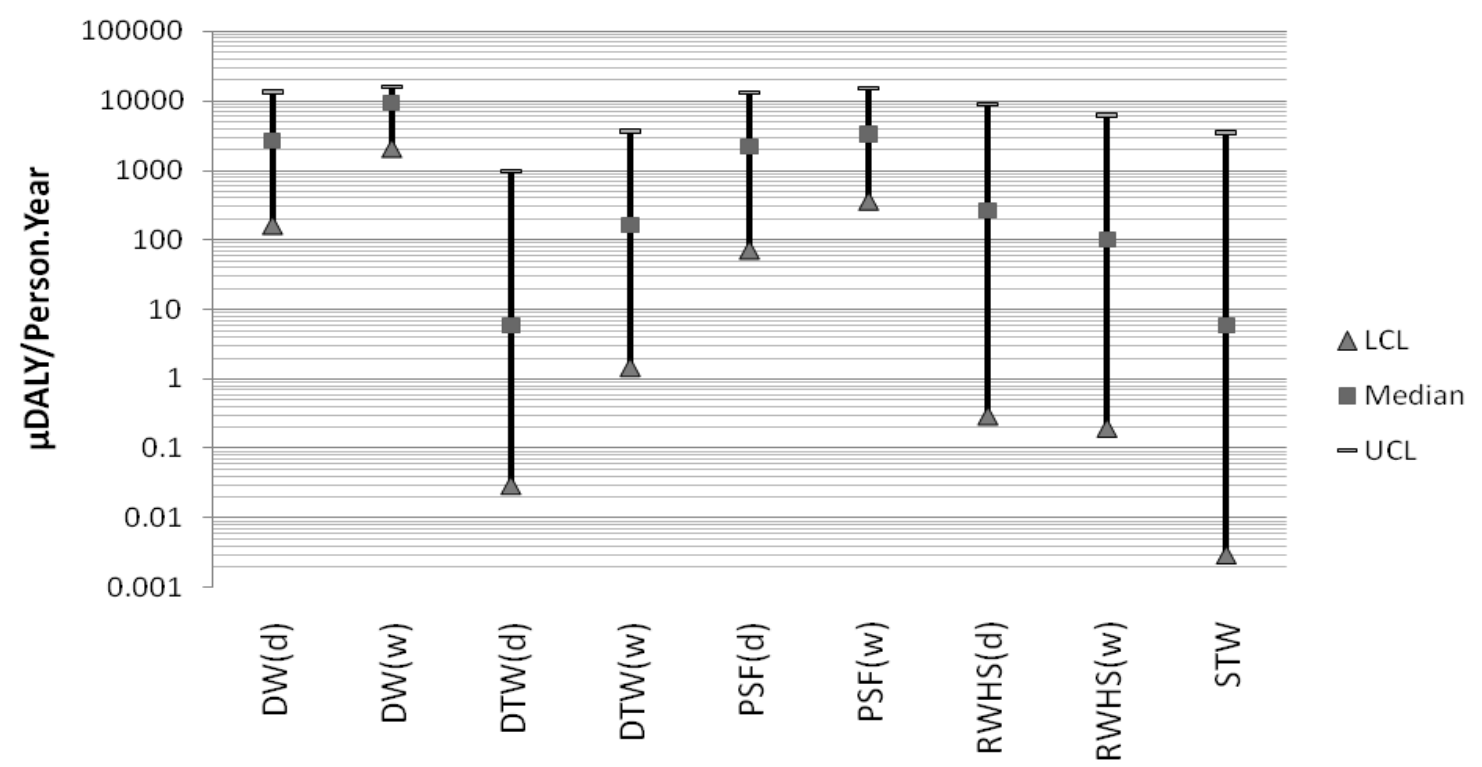

FIG. 4. Median, lower 5\% and upper 95\% disease burden and 90\% coincidence interval of water supply options in rural Bangladesh

effects from the many hazards will be much greater than $1 \mu \mathrm{DPY}$.

Disease burden estimates predicted using QMHRA models are based on evidence in the published literature, limited local data in Bangladesh, information and expert knowledge captured as numerical values based on judgment and extrapolation. There are both uncertainty and variability that combine to create inevitably broad confidence intervals in model predictions when using the model. In some studies it is sufficient to predict a reasonable estimate of the most likely levels of risk to be experienced using only the most likely or central statistics, or other representative values. It is important to carefully consider the context and the validity of all assumptions when applying the model. It is necessary to tailor the model assumptions to the country context. The model needs refinement and improvement as more reliable information become available. However, disease burden estimation by quantitative microbial risk assessment does not replace epidemiology, but the two approaches to health assessment are complementary.

\section{CONCLUSIONS}

The disease burden estimation by quantitative microbial health risk assessment has proved to be a useful tool for comparing the potential burden of microbial diseases associated with ingestion of microbial pathogens in drinking water from different sources. This assessment provides essential information like relative health risks of different health outcomes associated with water quality parameters and water from different sources, quantifiable improvement and residual health risk by different interventions and delineation of most cost-effective interventions in water supplies to reduce disease burden. The errors in model estimates arise from scarcity of epidemiological data in Bangladesh. The model needs further refinement by incorporating more accurate data and assumptions as new information become available in future.

\section{REFERENCES}

1. Acharjee, M., F. Jahan, F. Rahman, and R. Noor. 2013. Bacterial proliferation in municipal water supplied in mirpur locality of Dhaka city, Bangladesh. Clean - Soil, Air, Water 41: 1-8.

2. Ahmed, T., et al. 2013. Microbiological study of drinking water: qualitative and quantitative approach. Asian J. Microbiol. Biotech. Env. Sc. 15 (4): 23-30.

3. Marjan, S., et al. 2012. A comparative study ion Escherichia coli isolates from environmental and clinical samples. Bangladesh Journal of Microbiology 29 (2): 44-48.

4. Munshi, S. K., M. M. Rahman, and R. Noor. 2012. Detection of virulence potential of diarrheagenic Escherichia coli isolated from surface water of rivers surrounding Dhaka City. J. Bangladesh Acad. Sci. 36 (1):109-121.

5. World Health Organization. 2000-2012. Health statistics and information system: Estimates for 2000-2015. Available at: www.who.int/healthinfo/global_burden_disease/estimates/en/index $2 . h t m l$. Accessed 5 November, 2014.

6. World Health Organization. 2011. Guidelines for drinking-water quality, $4^{\text {th }}$ ed. World Health Organization, Geneva.

7. Gerba, C. P., J. B. Rose, C. N. Haas, and K. D. Crabtree. 1996. Waterborne rotavirus: a risk assessment. Wat. Res. 30: 2929-2940.

8. Ward R. L., D. I. Berstein, and E. C. Young. 1986. Human rotavirus studies in volunteers of infectious dose and serological response to infection. J. Infect. Dis. 154: 871-877.

9. ICDDR,B. 2003. International Centre for Diarrhoeal Diseases Research, Bangladesh Surveillance Update. Health and Science Bulletin 1:17-19.

10. Teunis, P. F. M., G. J. Medema, L. Kruidenier, and A. H. Havelaar. 1997. Assessment of the risk of infection by Cryptosporidium or Giardia in drinking water from a surface water source. Wat. Res. 31: 1333-1346.

11. Holcomb, D. L., et al. 1999. Comparison of six dose-response models for use with food-borne pathogens. Risk Anal. 19: 1091-1100.

12. Levine, M. M., et al. 1973. Pathogenesis of Shigella Dysenteriae 1 (Shiga) Dysentery. J. Infect. Dis. 127: 261-269

13. Messner, M. J., C. T. Chappell, and P. C. Okhuysen. 2001. Risk assessment for Cryptosporidium: a hierarchical Bayesian analysis of human dose response data. Water Res. 35: 3934-3940

14. DuPont, H. L., et al. 1995. The infectivity of Cryptosporidium parvum in healthy volunteers. N. Eng. J. Med. 332: 855-859. 
15. Okhuysen, P. C., et al. 1999. Virulence of three distinct Cryptosporidium parvum isolates for healthy adults. J. Infect. Dis. 180: 1275-1281.

16. Chappell, C. L., et al. 1999. Infectivity of Cryptosporidium parvum in healthy adults with pre-existing anti-C. parvum serum immunoglobulin G. Am. J. Trop. Med. Hyg. 60: 157-164.

17. Havelaar, A. H., and J. M. Melse. 2003. Quantifying public health risk in the WHO guidelines for drinking-water quality. Report No. 734301022/2003. RIVM, Bilthoven.

18. Murray, C. J. L, and A. D. Lopez. 1996. The Global burden of disease: A comprehensive assessment of mortality and disability from diseases, injuries and risk factors in 1990 and projected to 2020. Harvard: Harvard School of Public Health.
19. Watanabe, C., et al. 2004. Water intake in an Asian population living in arsenic-contaminated area. Toxicol Appl Pharmacol 198: 272-282.

20. Bangladesh Bureau of Statistics (BBS). 2012. Statistical year book Bangladesh Bureau of Statistics, Ministry of Planning, Government of Bangladesh.

21. Howard, G., et al. 2007. Disease burden estimation to support policy decision-making and research prioritization for arsenic mitigation. J. Water Health 5 (1): 67-81

22. Howard, G., et al. 2006. Risk assessment of arsenic mitigation options in Bangladesh. J. Health Popul. Nutr. 24 (3): 346-56. 